\title{
BORDERLINE GIANT CELL TUMORS (GCT) OF PROXIMAL TIBIAL EPIPHYSIS - ONCO-ORTHOPEDIC MANAGEMENT BETWEEN POSSIBILITIES AND LIMITS
}

doi: $10.2478 /$ rojost-2018-0063

\author{
R.S. Cismașiu ${ }^{1,2}$, R.M. Bîrluțiu ${ }^{1,3}$, A.Tudor ${ }^{1}$, D. Pop ${ }^{1,2}$, C.I. Stoica ${ }^{1,2}$ \\ ${ }^{1 " F o i s ̦ o r " ~ C l i n i c a l ~ H o s p i t a l ~ o f ~ O r t h o p a e d i c s, ~ B u c h a r e s t, ~ R o m a n i a ~}$ \\ 2"Carol Davila" University of Medicine and Pharmacy, Bucharest, Romania \\ 3"Victor Papilian" Faculty of Medicine, "Lucian Blaga"University, Sibiu, Romania
}

Intralesional tumoral procedures in giant cell tumor (GCT) are quoted as having a recurrence rate of up to $60 \%$. Thus, a number of studies suggest that broad resection is associated with a lower local recurrence risk compared to intralesional curettage, increasing the free recurrence interval from $84 \%$ to $100 \%$.

The TCG involvement of the proximal tibia occupies a particular place through the relationship with the articular line, but especially through the frequent, direct, or indirect interest of the extensor mechanism of the knee.

Purpose of the paper. Based on the unique tumor registry of "Foişor" Orthopedic Clinic, we proposed to follow all the cases of TCG with proximal tibial localized operation, beneficiaries of an "en bloc" resection and modular tumor prosthesis reconstructions - registering a number of 5 cases between 2009 and 2017.

Material and method. The initial evaluation was performed by radiography, CT-scan and MRI investigations and recurrence cases in which histopathological reassessment became mandatory after post-intralesional techniques were also included in the study.

The surgical technique followed tumor resection, tumor reconstruction, and the reconstruction procedure of the extensor of the knee, which involved the modulation of the modular tumor prosthesis with a rotary reversible flap of the medial gastrocnemius with the ankle of the patellar tendon to the prosthesis and flap.

Quantification of functional outcomes required the use of the revised Muscle-Skeletal Society Score (rMTS) and postoperative complications were centralized; the most commonly reported being the peroneal nerve palsy.

Results and discussions. The results obtained were compared to the orthopedic oncology literature data for such a procedure, following their superposition and discrepancy.

The challenge of such an orthopedic oncology intervention is the reconstruction of the extensor, with definite functional implications of limiting the knee extension, as well as avoiding the peroneal nerve palsy.

However, the results can be reproducible, with an absent relapse rate.

Keywords: giant cell tumor, resection-tumor reconstruction, gastrocnemius medial flap 